\title{
An Alternative Corrosion Resistance Test Method for Solar Cells and Interconnection Materials Limiting the Number of Long-lasting and Expensive Damp-Heat Climate Chamber Tests
}

\author{
Bas B. Van Aken, Robert J. Gouwen ", Dirk Veldman\#, Evert Bende and Wilma Eerenstein \\ ECN - Solar Energy, P.O. Box 1, 1755 ZG Petten, the Netherlands \\ *Present address: Shell Nederland - Materials and Corrosion, P.O. Box 3000, 3190 GA Rotterdam, the \\ Netherlands \\ ${ }^{\#}$ Present address: Philips Group Innovation - Research, High Tech Campus 4 1.4.18, 5656 AE Eindhoven, \\ the Netherlands
}

\begin{abstract}
Damp-heat testing of PV modules is a timeconsuming process, taking months. We present an alternative test method: electrochemical noise (EcN) measurements. Data acquisition times vary between minutes for direct exposure to several tens of hours for encapsulated samples. EcN measurements are presented for several solar cell concepts and different environments. We have found that the degradation in damp-heat testing is proportional to the electrochemical noise signal. In conclusion, the electrochemical noise measurements are a fast, versatile tool to test the corrosion resistance of solar cells, which can be tested for different environments including encapsulation.

Index Terms - climate testing, corrosion, experimental set-up, photovoltaic cells, silicon.
\end{abstract}

\section{INTRODUCTION}

Acetic acid release by the most commonly applied encapsulant EVA, in combination with heat and humidity, leads to degradation of the metallisation on crystalline Si solar cells, see for an overview [1]. However, damp-heat testing is a time-consuming process as a full IEC cycle requires 1000 hours (6 weeks). We present an alternative, fast test method for the corrosion sensitivity of metal/wafer combinations.

Electrochemical noise measurements are a standard tool in the metal industry to evaluate the corrosion rates and corrosion resistances of steel and aluminium alloys. Although the theoretical basis is not completely developed, the EcN measurements can be related to the corrosion rate. In particular, the electrochemical resistance noise has a good inverse correlation with the corrosion sensitivity. The electrochemical current noise is also rather consistent with the corrosion susceptibility. [2]

The noise consists of potential and/or current fluctuations generated by spontaneous corrosion reactions. The EcN measurement is sensitive to initiation and propagation of corrosion. The electrochemical current noise is proportional to the corrosion activity and the loss of metal.

EcN measurements are presented for several situations. For testing variations in MWT cell technology, we varied both the metallisation pastes used and the area of the overlap region between $\mathrm{Ag}$ and $\mathrm{Al}$ in dedicated test structures. It was found that the larger the Ag/Al overlap area, the larger the EcN signal is. Also, when we use metallisation pastes that show little degradation in damp-heat testing, the current noise is small and vice versa.

We have also tested the corrosion rate of the front side and back side metallisation of n-type monocrystalline solar cells [3]. The current noise shows a clear bias towards corrosion occurring on the front side.

\section{EXPERIMENTAL SET-UP}

The set-up exists of two pieces of sample material. These are placed on either side of a tube which is filled with an electrolyte. The two pieces are connected to the electronics unit, recording the potential and the current flow between the samples. The two samples can be placed as a conventional galvanic cell, with a working electrode and a counter electrode. The observed electrochemical current between the two electrodes could give information on the corrosion rate.

Alternatively, for noise measurements, we actually place two identical samples with the same composition towards the electrolyte. In this way, there should be no electrochemical potential or current. However, depending on the combination of samples and environment, corrosion can still occur. Every corrosion event on either of the electrodes will lead to a small, short current peak. The sign of the peak is determined by the reaction and electrode. These current peaks are observed as electrochemical noise.

Historically, this noise on the current and potential data was regarded as a nuisance, but nowadays it is well-known that the noise contains valuable information. The current noise is most sensitive to the corrosion rate. To extract this information, first the DC trend in the current noise was removed. Sometimes the raw current noise data is already enough to determine differences in corrosion resistance. If a qualitative ranking of the electrochemical behaviour is required, the noise 
data, i.e. the current peaks in the time domain, are transformed to the frequency domain, i.e. the number of times each current peak value occurs.

The electrolyte can be chosen to simulate the conditions that a solar cell is exposed to in a module. For instance, artificial rain water or an acetic acid solution to mimic degraded EVA.

\section{RESULTS}

\section{A. MWT Cells in Artificial Rain Water}

Climate chamber tests at $85 \%$ humidity and $85^{\circ} \mathrm{C}$ are standard tests to qualify the reliability of modules. The IEC criterion is that a module should have no more than $5 \%$ power loss after 1000 hours damp-heat exposure. Moreover, module manufacturers expect modules to survive exposure for at least twice as long and still have less than $5 \%$ power loss. However, 2000 hours of damp-heat testing takes 12 weeks, without breaks for intermediate measurements. We have fabricated MWT modules with different metallisation pastes A and B. After 500 hrs exposure, there is no difference between the two series of modules in power loss. After 1000 hours, that is 6 weeks exposure, modules $B$ fail catastrophically with $25 \%$ and more power loss. In contrast modules with paste A have only 2- 3\% power loss after 2500 hours, but this is only apparent after more than 3 months of exposure. We measured the electrochemical noise of these cell types as described above.

Fig. 1 shows the current noise of the MWT solar cells, described above, exposed to artificial rain water - a common corrosion promoting agent - in the electrochemical noise setup.

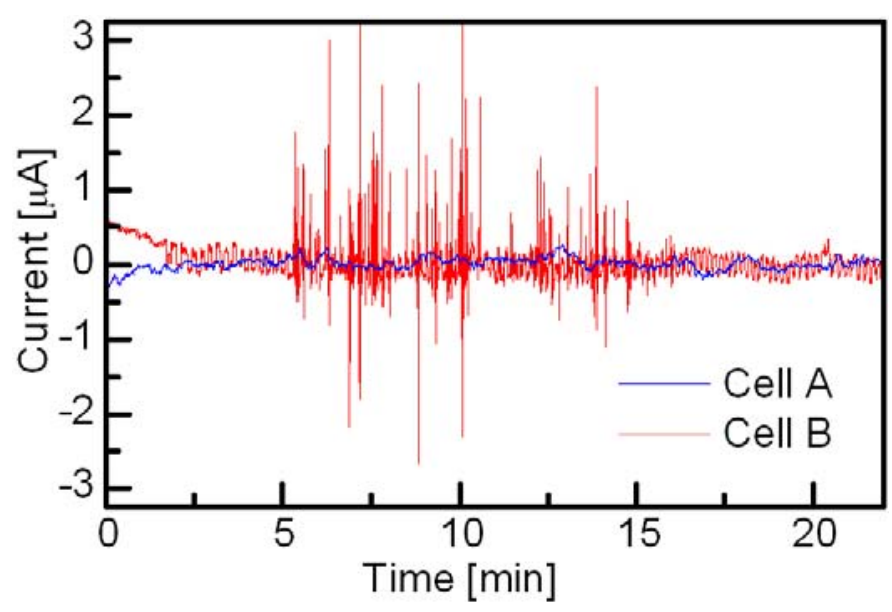

Fig. 1. Electrochemical current noise as a function of time for two MWT cells with different base contacts. The DC trends in the current noise signals have been removed.
Cell type A shows hardly any noise signal during the 25 minutes of this test. Cell type B shows a more interesting behaviour. Initially there is hardly any noise signal too, up to 2 minutes. Then we see a sudden increase in noise signal with peaks of about $0.3 \mu \mathrm{A}$. The much lower noise signal for the first few minutes indicates a low initiation rate for corrosion. However, after 5 minutes the noise increases sharply in intensity, displaying strong peaks of up to $3 \mu \mathrm{A}$. This regime continues for about 10 minutes. Then the noise signal fades to the lower values again, but still higher than in cell type A.

We have shown that modules with cell type A show hardly any damp-heat degradation and cell type A shows a very low noise signal. In stark contrast, cell type B shows a much larger noise signal and modules with cell type B fail the 5\% power loss criterion after 1000 hours damp-heat exposure. The damp-heat experiments take 6 to 15 weeks, whereas the EcN measurement takes only half an hour.

\section{B. Silver/Aluminium Overlap Regions}

Corrosion can occur when two metals are in contact. This situation occurs, for instance, at the rear side of p-type solar cells, where the Al BSF is contacted with Ag paste for interconnection purposes. In most cases, not only the metal/metal/electrolyte combination determines the corrosion activity but also the geometry. We have fabricated dedicated test structures to test the influence of the metallisation paste formulation (not shown) and of the amount of $\mathrm{Ag} / \mathrm{Al}$ overlap area. The three samples have been exposed to $0.01 \mathrm{M}$ acetic acid solution to simulate degraded EVA and the electrochemical noise has been recorded for a period of about an hour.

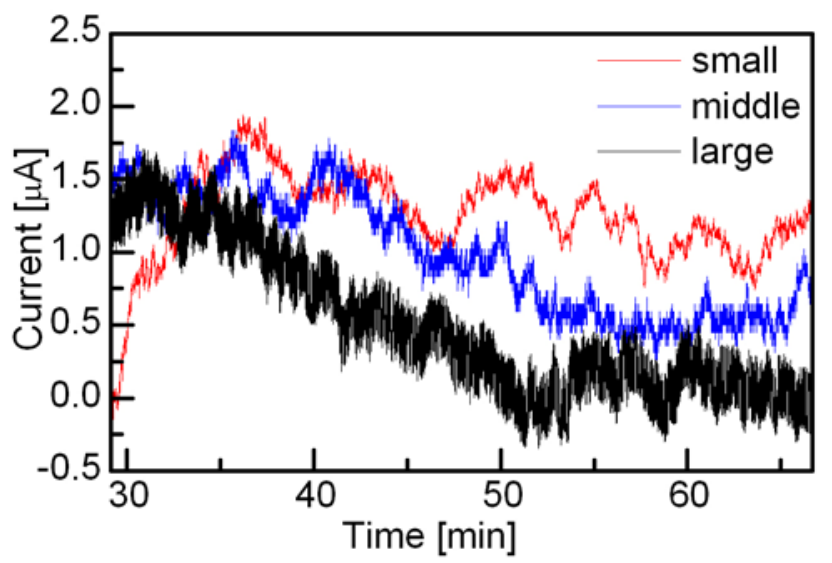

Fig. 2. Electrochemical current noise against time for the three Ag/Al overlap samples. The DC trends in the current noise signals have not been removed to be able to see all three curves.

Fig. 2 shows the current noise transients for the three samples with small, middle and large overlap areas. Clearly, 
the noise level increases with overlap area. The samples have been inspected visually after the EcN measurement. Samples with low noise signal, like the "small overlap" sample shown in Fig. 2 (red), appear similar to non-exposed samples. In contrast, the sample with the same metal paste but large overlap area, shown in black in Fig. 2, looks strongly degraded with uneven surfaces and blurred edges. This suggests that the larger signal for "large" overlap area is not only due to the larger area but also that the corrosion has advanced further.

\section{C. n-Type Pasha Front and Rear Side}

In n-type H-pattern solar cells, the front side metallisation has to make contact with the p-type emitter through the passivation layer, typically SiNx. The rear side metallisation contacts the $\mathrm{n}^{++-B S F}$ surface. To ensure a well conducting contact with the front side, a few percent $\mathrm{Al}$ is added to the Ag paste. The back side metallisation has only Ag as metallic component. In a typical module the n-type cells are encapsulated with EVA. It is well known that under dampheat conditions the EVA can degrade and form acetic acid. [1]

We have placed the front and back side of such a solar cell in contact with an electrolyte, consisting of a $0.01 \mathrm{M}$ acetic acid solution to mimic the corrosive behaviour of degraded EVA. The current data is plotted against time in Fig. 3. Initially we observe a current of several $\mu \mathrm{A}$, indicating a relatively large difference in electrochemical potential. After time, the current falls off to $200 \mathrm{nA}$. In the magnified inset, we can clearly observe that the "negative" noise signal is much larger than the noise peaks in the positive direction.

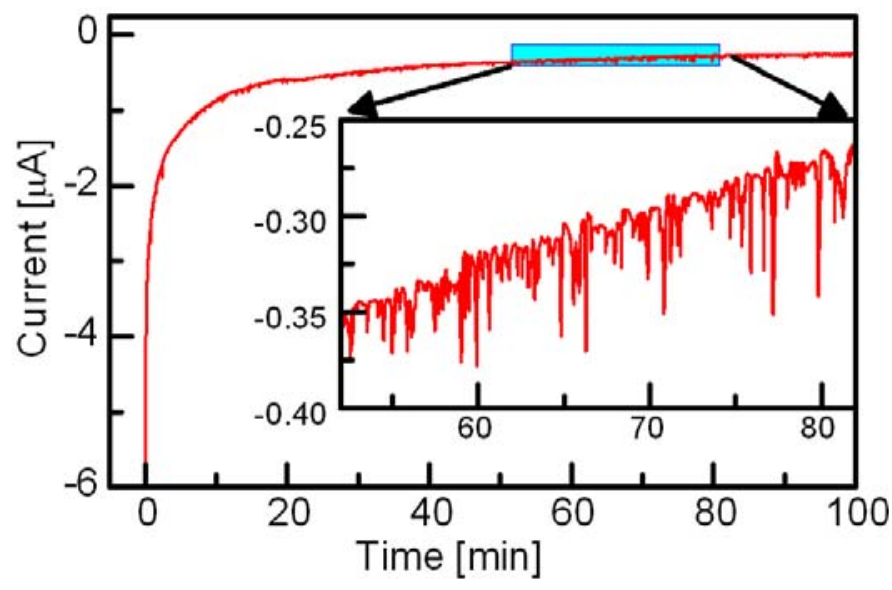

Fig. 3. Electrochemical current for a conventional galvanic cell-type measurement of an n-type H-pattern wafer. The inset shows a magnification of a part of the noise signal.
Fig. 4 shows the EcN measurements for the front and back of this solar cell in the "noise signal set-up". Clearly, the back side data shows a much lower noise than the front side, in agreement with the observations in the "galvanic cell set-up". Post-mortem analysis of damp-heat exposed laminates with EVA and these solar cells also show corrosion damage on the front. In contrast, on the rear and in laminates with non-EVA encapsulant no corrosion and also no degradation in fill factor is observed after 2000 hours of damp-heat testing.

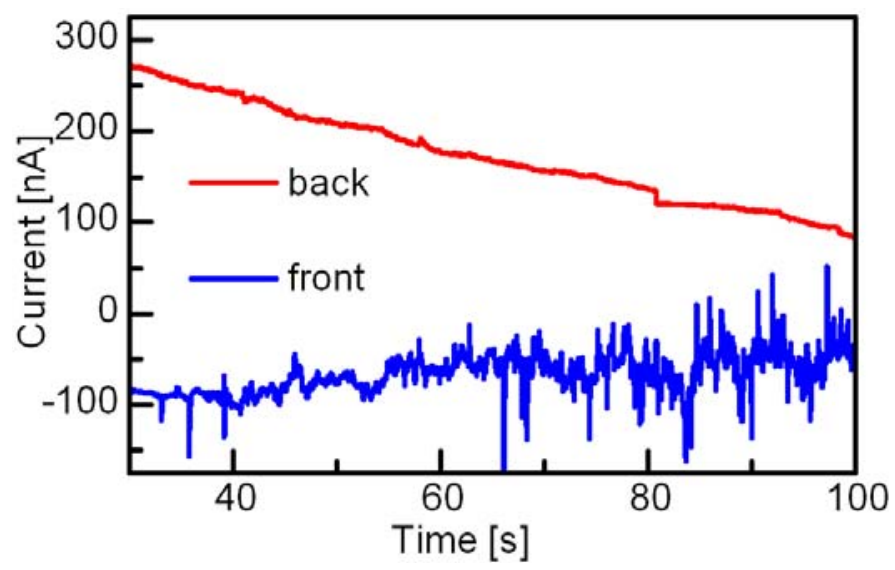

Fig. 4. Electrochemical current noise for two front side (blue) and two back side (red) grids.

\section{CONCLUSIONS}

We have presented corrosion tests of solar cells using electrochemical noise measurements $(\mathrm{EcN})$. EcN is a fast and direct corrosion measurement technique, whereby results can be obtained within one hour. It correlates with $\mathrm{DH}$ degradation mechanisms involving corrosion, which is rather common in EVA-encapsulated crystalline $\mathrm{Si}$ modules. Furthermore, the EcN test can be done as an evaluation tool when probing alternative brands, formulations or processing for metallisation paste and as a screening test for new batches of metallisation paste.

\section{REFERENCES}

[1] P. Hacke, "Research opportunities in reliability of photovoltaic modules", in Workshop catalysing innovation in $P V$ manufacturing, Golden, CO, 2010.

[2] R.A. Cottis and S. Turgoose, "Electrochemical noise measurements - a theoretical basis", Materials Science Forum, vol. 192-194, pp. 663-672, 1995.

[3] I.J. Bennett and B.B. Van Aken, "Testing compatibility of ntype cells and module materials", in 2nd n-PV workshop, Amsterdam, the Netherlands, 2012. 